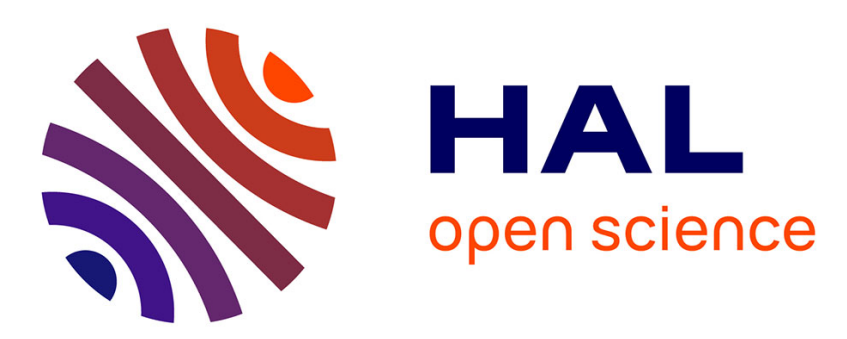

\title{
Why are some oxides metallic, while most are insulating?
}

Jerry B Torrance, P. Lacorre, Chinnarong Asavaroengchai, Robert M Metzger

\section{To cite this version:}

Jerry B Torrance, P. Lacorre, Chinnarong Asavaroengchai, Robert M Metzger. Why are some oxides metallic, while most are insulating?. Physica C: Superconductivity, 1991, 182 (4-6), pp.351-364. 10.1016/0921-4534(91)90534-6 . hal-02196091

\section{HAL Id: hal-02196091 \\ https://hal.science/hal-02196091}

Submitted on 26 Jul 2019

HAL is a multi-disciplinary open access archive for the deposit and dissemination of scientific research documents, whether they are published or not. The documents may come from teaching and research institutions in France or abroad, or from public or private research centers.
L'archive ouverte pluridisciplinaire HAL, est destinée au dépôt et à la diffusion de documents scientifiques de niveau recherche, publiés ou non, émanant des établissements d'enseignement et de recherche français ou étrangers, des laboratoires publics ou privés. 


\title{
Why are some oxides metallic, while most are insulating?
}

\author{
Jerry B. Torrance, ${ }^{1}$ Philippe Lacorre, ${ }^{1}$ Chinnarong Asavaroengchai, ${ }^{2}$ Robert M.Metzger ${ }^{2}$ \\ ${ }^{1}$ IBM Research Division, Almaden Research Center, 650 Harry Road, San Jose, CA 95120-6099, USA \\ ${ }^{2}$ Department of Chemistry, University of Alabama. Tuscaloosa, AL 35487, USA
}

\begin{abstract}
A large variety of undoped binary and ternary transition metal oxides, including formally divalent, trivalent and tetravalent metal cations, have been examined. These 76 compounds are classified as either "metals", "insulators", or having a "metal-to-insulator" transition. In an attempt to understand these variations, the Zaanen-Sawatzky-Allen framework was used in which each compound can be characterized by three parameters: the Coulomb correlation or disproportionation energy $\left(U^{\prime}\right)$, the charge-transfer energy $(\Delta)$ and the bandwidth $(W)$. Assuming $W$ is constant, we have calculated $U^{\prime}$ and $\Delta$ using a simple ionic model, which includes only the gas phase ionization potentials and the bare electrostatic Coulomb interactions between the ions. With this model, the occurrence of metallic conductivity is remarkably well accounted for in these oxides.
\end{abstract}

\section{Introduction}

In some oxides of bismuth and copper, superconductivity has been discovered [1-3] at relatively high temperatures. One approach to understanding the electronic properties of these materials is to focus on a few representative examples and to examine them in great depth. An alternative and complementary strategy is to view these oxides in the broader context of the other transition metal oxides and to attempt to understand the differences between them. Following this latter approach, one is immediately faced with having to understand the large differences in their conductivity behavior: the majority of these undoped oxides are insulating, some exhibit metallic conductivity, but very few are superconducting. The first step in this strategy is thus to understand what basic difference between these oxides is responsible for the variety of their conductivity behavior; i.e., why are some undoped oxides metallic, whereas the majority are insulating? If we can find a way to understand this, we can continue and consider doped systems, with considerable degree of confidence in our starting point. It could also be argued that this is a more reliable strategy to arrive at the fundamental electronic energies for these oxides, which underlie the basic assumptions for the various theories for high-temperature superconductivity.

The conductivity of a large number of simple and perovskite oxide compounds has been compiled in several review articles [4-9]. In table 1 we have summarized [10] some of the data for 76 undoped systems [11] involving transition metals (including rare earths) in divalent (II), trivalent (III), and tetravalent (IV) formal oxidation states [9]. Here the conductivity behavior of these oxides has been crudely and arbitrarily divided into "metals" and "insulators" on the basis of the magnitude (and not the temperature dependence) of their conductivity, $\sigma(300 \mathrm{~K})$, at room temperature: "metals" are defined as having $\sigma(300 \mathrm{~K})>1 \mathrm{~S} / \mathrm{cm}$, while "insulators" have $\sigma(300 \mathrm{~K})<1 \mathrm{~S} / \mathrm{cm}$. In a few cases (for example, $\mathrm{FeO}, \mathrm{SnO}_{2}$, and $\mathrm{In}_{2} \mathrm{O}_{3}$ ), the conductivity is high because of deviations from ideal stoichiometry, but their intrinsic "insulating" behavior is evident from optical measurements [12,13]. A few oxides have metal-insulator transitions $[5,14-16]$ and are so labeled in table 1. 
Table 1

Conductivity of undoped transition metal oxides

\begin{tabular}{|c|c|c|c|c|c|c|c|c|}
\hline & \multicolumn{2}{|c|}{ Divalent } & \multicolumn{3}{|l|}{ Trivalent } & \multicolumn{3}{|c|}{ Tetravalent } \\
\hline & MO & $\mathrm{La}_{2} \mathrm{MO}_{4}$ & $\mathrm{M}_{2} \mathrm{O}_{3}$ & $\mathrm{LaMO}_{3}$ & $\mathrm{LaSrMO}_{4}$ & $\mathrm{MO}_{2}$ & $\mathrm{SrMO}_{3}$ & $\mathrm{Sr}_{2} \mathrm{MO}_{4}$ \\
\hline $\mathrm{Ti}$ & met. & & met./ins. & met./ins. & & ins. & ins. & ins. \\
\hline V & met. & & met./ins. & ins. & ins. & met./ins. & met. & ins. \\
\hline $\mathrm{Cr}$ & & & ins. & ins. & ins. & met. & met. & \\
\hline $\mathrm{Mn}$ & ins. & & ins. & ins. & ins. & met. & ins. & ins. \\
\hline $\mathrm{Fe}$ & ins. & & ins. & ins. & ins. & & met. & \\
\hline $\mathrm{Co}$ & ins. & ins. & & ins. & ins. & & met. & \\
\hline $\mathrm{Ni}$ & ins. & ins. & & met./ins. & met. & & & \\
\hline $\mathrm{Cu}$ & ins. & ins. & & met. & met. & & & \\
\hline $\mathrm{Nb}$ & met. & & & & & ins. & & \\
\hline Mo & & & & & & met. & met. & met. \\
\hline $\mathrm{Ru}$ & & & & met. & & met. & met. & met. \\
\hline $\mathbf{R h}$ & & & ins. & ins. & & met. & & \\
\hline Sn & ins. & & & & & ins. & ins. & ins. \\
\hline $\mathbf{L a}$ & met. & & ins. & & & & & \\
\hline $\mathrm{Ce}$ & met. & & ins. & & & ins. & ins. & \\
\hline $\operatorname{Pr}$ & met. & & ins. & & & ins. & ins. & \\
\hline $\mathrm{Nd}$ & met. & & ins. & & & & & \\
\hline $\mathrm{Eu}$ & ins. & & ins. & & & & & \\
\hline $\mathrm{Yb}$ & ins. & & ins. & ins. & & & & \\
\hline
\end{tabular}

In order to understand the differences between the "metals" and "insulators" in table 1, one might first be tempted to try an elementary electron band structure approach. According to the most basic feature of this approach, compounds in which the transition metal cation has an odd number of electrons would be predicted to be metallic, whereas those with an even number of electrons might be insulating. There are 36 oxides in table 1 with an odd number of electrons, but most of these (22) are "insulating"; there are 40 oxides with an even number of electrons, but 14 of these are "metallic". Thus, the simplest aspect of band structure theory appears not to be a promising starting point to account for the conductivity behavior exhibited in table 1 . Of course, more sophisticated band structure calculations should be used to examine each of these compounds in detail. However, such calculations are not available for all the oxides, and there are serious questions whether these calculations would effectively include the effects of Coulomb correlations, believed to be often of critical importance [4-9].

\section{Ionic model}

A simple, and yet powerful, framework which includes correlation effects has been introduced and developed by Zaanen, Sawatzky and Allen (ZSA) [17,18]. According to this picture, oxides (as well as halides, sulfides, etc.) can be described in terms of the relative energies of three electronic energy states near the Fermi level, as shown in fig. 1. The fully occupied oxygen $2 p$-states are shown as the shaded band on the left side of the vertical energy axis, at the same energy for each of the five different examples shown. On the right side of the vertical energy axis and shown unshaded is the lowest unoccupied metal orbital (corresponding to the metal conduction band) which lies at an energy $\Delta$ above the former. $\Delta$ is seen to increase in going toward the right in fig. 1. This unoccupied metal orbital lies at an energy $U^{\prime}$ above the highest occupied (shaded) metal orbital, as shown in fig. 1 . In order to be sure to include the correlations, we use semi-localized orbitals or energy levels (as opposed to bands) which each holds one electron (not two). The prime on $U^{\prime}$ reminds us that this energy (defined in fig. 1) is not always the Hubbard [4,5] $U$. Note also that the 


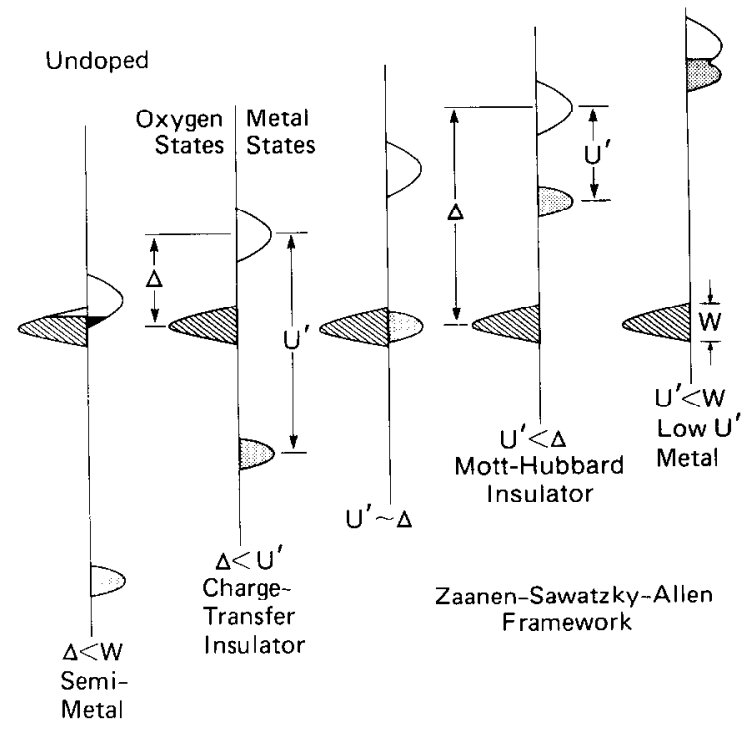

Fig. 1. A schematic diagram of the relative energies of the oxygen and metal states in the ZSA framework. Five examples are shown with different relative values of $\Delta, U^{\prime}$ and $W$ (which are also defined in the figure).

energies $\Delta$ and $U^{\prime}$ in fig. 1 are measured between the centers of the orbitals. In the solid, the electronic overlap between orbitals broadens them to a width, $W$, which is assumed here to be the same for each of these three states, for simplicity. Any given compound may then be represented by its values [19] of $U^{\prime}, \Delta$, and $W$.

The different cases in fig. 1 can be conveniently discussed in the $U^{\prime}-\Delta$ space of a Zaanen-SawatzkyAllen diagram, as shown in fig. 2. For the case of $W<\Delta, U^{\prime}$, the occupied orbitals in fig. 1 do not overlap the unoccupied orbital and the compounds are insulating. ZSA $[17,18]$ distinguish two different types of insulators:

(1) Charge-transfer insulators when $W<\Delta<U^{\prime}$ (left of fig. 1 and upper-left of fig. 2). In this case, the gap $\sim(\Delta-W)$ is dominated by the value of $\Delta$; and

(2) Mott-Hubbard insulators, when $W<U^{\prime}<\Delta$ (right of fig. 1 and lower-right of fig. 2). Here the gap $\sim\left(U^{\prime}-W\right)$ is dominated by $U^{\prime}$.

Corresponding to these two types of insulators, there are two distinct types of optical excitations. These have a clearer physical interpretation in the localized limit (i.e., $W \ll \Delta, U^{\prime}$ ), where the first is an

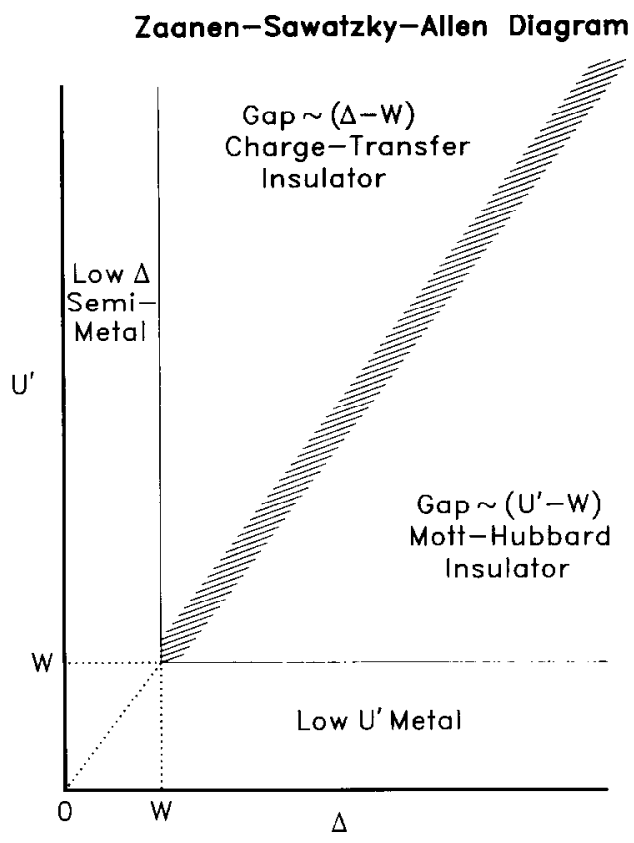

Fig. 2. The ZSA diagram showing the different regions that compounds may lie in, depending on their values of $U^{\prime}$ and $\Delta$.

oxygen-to-metal charge-transfer transition at an energy 4 , and the second is a Hubbard-like metal-tometal charge-transfer transition at an energy $U^{\prime}$, which corresponds to the disproportionation energy. In charge-transfer insulators, the former is the lowest lying excitation and dominates the gap, whereas the Hubbard-like transitions are lowest in the MottHubbard type of insulators. A metal results when either of these two gaps approaches zero and hence there exist two types of correlated electron metals:

(1) "Low- $\Delta$ metals", when $\Delta<W$ (far left of figs. 1 and 2), in which the lowest-lying metal conduction band overlaps the occupied oxygen $2 p$-valence states; and

(2) "Low- $U^{\prime}$ metals", when $U^{\prime}<W$ (far right of fig. 1 and bottom of fig. 2), in which case the two metal orbitals merge to form a partially filled band.

The goal of this paper is to obtain values of $U^{\prime}$ and $\Delta$ for each of the 76 compounds in table 1 and examine them within the ZSA framework, by plotting them as in fig. 2. While reasonable experimental values for a few of these compounds are available, a comparison among all oxide systems is more appropriately carried out with a self-consistent set of ap- 
proximate values obtained in the same manner for each of them. Such a set may be obtained, if we approximate these oxides as ionic solids containing transition metal cations $\mathrm{M}^{\nu+}$ and oxide anions $\mathrm{O}^{2-}$, with negligible wave function overlap between them. Such simplc ionic models have proven useful for calculating ground state properties of oxides [20,21], including the significant role of the Madelung energy in high- $T_{\mathrm{c}}$ copper oxides [22-24]. The values of $U^{\prime}$ and $\Delta$ obtained in this approximation are called $U_{0}^{\prime}$ and $\Delta_{0}$, where the zero subscripts remind us that they are theoretically calculated values for the simple ionic model. Another reason for the subscripts is that both of these energies correspond to excitons. For example, the energy $U_{0}^{\prime}$ corresponds to the excitation of an electron from one transition metal cation to its nearest neighbor (at a distance $d_{M-M}$ ), and is given in terms of the difference between the ionization potential $I_{v+1}$ of $\mathrm{M}^{v+}$ and its electron affinity $A=I_{\nu}$ :

$U_{0}^{\prime}=I_{v+1}(\mathrm{M})-I_{v}(\mathrm{M})-e^{2} / d_{\mathrm{M}-\mathrm{M}}$.

The term $e^{2} / d_{\mathrm{M}-\mathrm{M}}$ is the Coulomb attraction between the excited electron and the hole left behind and is present because this is a local excitation (exciton). This interaction is not included in some definitions [19] of $U$, which correspond to exciting an electron to infinity and bringing it back to another (uncorrelated) metal site, i.e., an electron-hole excitation with no interaction between them. The latter definition is physically related to experiments of photoemission, while the former can be compared with optical measurements. (There are important differences in the screening of these two types of excitations, as we shall see below.)

Correspondingly, the energy $\Delta$ to excite an electron from $\mathrm{O}^{2-}$ to a neighboring transition metal (at a distance $d_{\mathrm{M}-\mathrm{O}}$ ) involves the difference between the ionization potential $I\left(\mathrm{O}^{2-}\right)$ of $\mathrm{O}^{2-}$ (which is equal to the electron affinity $\left.A\left(\mathrm{O}^{-}\right)\right)$and the electron affinity $\left(A=I_{v}\right)$ of $\mathrm{M}^{v+}$. In addition, there exists a term $\Delta V_{\mathrm{M}}$, the difference in electrostatic Madelung site potentials, that the electron experiences when it changes sites $[25,26]$ :

$$
\Delta_{0}=e \Delta V_{\mathrm{M}}+A\left(\mathrm{O}^{-}\right)-I_{v}(\mathrm{M})-e^{2} / d_{\mathrm{M}-\mathrm{O}},
$$

where the term $e^{2} / d_{\mathrm{M}-\mathrm{O}}$ includes the electron-hole attraction of the exciton. Again, the subscript zero on $\Delta_{0}$ reminds us that this is the energy calculated for an exciton using a simple ionic model.

In this very simple ionic model, the values for $U_{0}^{\prime}$ and $\Delta_{0}$ depend only on the electrostatic interactions between ions and the gas-phase ionization potentials, which are used as values of $I_{v}$. The electrostatic site potentials were taken from earlier calculations by Broughton and Bagus [27], were calculated using standard Ewald techniques [28], or were estimated from known calculations or for an approximate crystal structure. The values of $d_{\mathrm{M}-\mathrm{O}}$ and $d_{\mathrm{M}-\mathrm{M}}$ were obtained directly from the crystal structures. The gas-phase values for $I_{v}$ were generally [29] taken from experiment [30], while a value [31] of $-7.70 \mathrm{eV}$ was used for $A\left(\mathrm{O}^{-}\right)$.

\section{Closed-shell oxides}

Before discussing the results for these transition metal oxides, it is instructive to test these ideas and approximations on some simpler closed-shell oxides, such as those shown in table 2 . In these insulating compounds, the occupied metal orbitals are usually far below the Fermi energy and not involved, i.e., $U^{\prime} \gg \Delta$. Hence, there are only two relevant orbitals, separated by the energy $\Delta$ (left of fig. 1) and the optical band gap is given by $(\Delta-W)$, Using eqs. (1) and (2), we have calculated $U_{0}^{\prime}$ and $\Delta_{0}$ for these closed-shell oxides and included them in table 2. (It can be seen that the values of $U_{0}^{\prime}$ are indeed very large and in this case of closed shell metal cations $U_{0}^{\prime}$ does not correspond to a Hubbard-like $U$ ). In order to test the validity of this ionic model calculation, we can compare the experimentally measured values [13,32] of the optical band gap (listed in table 2) with the predicted gap $>\left(\Delta_{0}-W\right)$ by plotting the gap versus $\Delta_{0}$ in fig. 3. In general, the expected trend of larger gap with larger $A_{0}$ is observed. Three compounds, $\mathrm{Y}_{2} \mathrm{O}_{3}, \mathrm{CeO}_{2}$, and $\mathrm{SiO}_{2}$ (marked by filled boxes in fig. 3 ) appear to lie somewhat outside this trend, suggesting that they are less well described by such a simple ionic model. Treating these three as exceptions, the remaining data (shown as open boxes) are fitted to a straight line with slope $=1$.

The agreement is remarkable considering the approximations made. The gap extrapolates to zero at $\Delta_{0}=10.0 \mathrm{eV}$, considerably larger than estimates for 


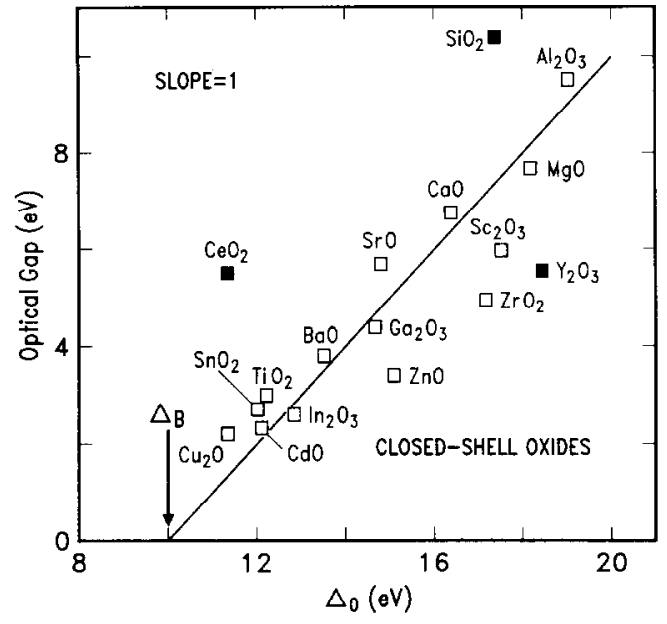

Fig. 3. The experimental optical energy gap of a series of simple oxides with closed-shell cations plotted as a function of the calculated value of the parameter $\Delta_{0}$. The straight line is a leastsquares fit to the open boxes, assuming a slope $=1$. The intercept $\Delta_{\mathrm{B}}=10.0 \mathrm{eV}$ indicates the value of $\Delta_{0}$ where the gap would go to zero.

$W$. But we must remember that, in this ionic model, we have not included any of the effects of the overlap between ions (covalency, hybridization, crystal field splittings, screening, electronic polarizability), or the motion of the ions (lattice relaxation, ionic polarizability). Our basic assumption is not that these effects are small; rather, that they are similar for all the oxides in tables 1 and 2, so that the differences in $U_{0}^{\prime}$ and $\Delta_{0}$ are dominated by the large differences in $I_{v}$ and $\Delta V_{M}$ via eqs. (1) and (2). The main consequence of these neglected effects is to reduce (or screen ) the actual value of $U^{\prime}$ and $\Delta$ below those calculated in table 2. Detailed calculations [33] on $\mathrm{La}_{2} \mathrm{CuO}_{4}$, for example, show that the electronic relaxation energy around an isolated hole $\left(\mathrm{O}^{-}\right.$or $\left.\mathrm{Cu}^{3+}\right)$ and that around an electron $\left(\mathrm{Cu}^{+}\right)$are comparable and are of the order of $\sim 3 \mathrm{eV}$. Thus, the energy to create a separated electron and hole is predicted [34] to be reduced (screened) by $\sim 6 \mathrm{eV}$, i.e., both $U$ and $\Delta$ are screened by this amount. On the other hand, in the local excitations $U_{0}^{\prime}$ and $\Delta_{0}$ considered here, the electron-hole pair is more like a dipole than two isolated charges and the electronic and lattice relaxation energies should be much smaller. If the screening in this case were, say, $\sim 3 \mathrm{eV}$, then the intercept in fig. 3 at $\Delta_{0}=10.0 \mathrm{eV}$ (unscreened) would correspond to $A \sim 7 \mathrm{eV}$ (screened), which is comparable (but somewhat higher) with estimates [3536 ] of the bandwidth. In conclusion, the magnitude of the intercept $\Delta_{0}=10.0 \mathrm{eV}$ in fig. 3 is not unreasonable.

The calculated values of $U_{0}^{\prime}$ and $\Delta_{0}$ are also considerably larger than the values of $U$ and $\Delta$ obtained from spectroscopy. For example, in the case of $\mathrm{NiO}$, photoemission experiments [17] give an estimate of $U \sim 7-8 \mathrm{eV}$, while a value of $U_{0}^{\prime}=12.2 \mathrm{eV}$ is calculated below. It is important to recognize that these values should not be directly compared because of the basic difference in their definition and physical meaning, and the corresponding differences in screening. As mentioned above, the photoemission $U$ corresponds to exciting a separated electron and hole on the Ni-sites, which would have an unscreened ionic model energy of $I_{3}(\mathrm{Ni})-I_{2}(\mathrm{Ni})=17.0 \mathrm{eV}$. These separated charges get strongly screened and together with covalent effects presumably reduce the above estimate down to the experimental values of $U \sim 7-8 \mathrm{cV}$. The cxcitation corresponding to $U_{0}^{\prime}$, on the other hand, corresponds to exciting the electron and hole on neighboring Ni-sites. This energy in the ionic model (eq. (1)) is reduced by the Coulomb attraction between them from $17.0 \mathrm{eV}$ to an unscreened estimate of 12.2 $\mathrm{eV}$. This calculated value of $U_{0}^{\prime}$ will be reduced by screening and covalent effects differently than the $U$ excitation. Thus, it is difficult to compare the values of $U$ and $U_{0}^{\prime}$ and more difficult to use this comparison to estimate the crudeness of the oversimplified ionic model. The basic assumption of this paper is that the important screening and covalent effects not included in the ionic model may be large, but will be sufficiently similar in magnitude for all the oxides considered, so that the major variations in $U_{0}^{\prime}$ and $A_{0}$ will be dominated by the variations in $I_{v}$ and $\Delta V_{\mathrm{M}}$.

The fact that the optical gap for closed-shell oxides extrapolates to zero for low $\Delta_{0}$ is interpreted here as indicating that compounds with $\Delta_{0}<10 \mathrm{eV}$ would be metallic. Similarly, we interpret the transition between insulators and metals in the ZSA diagram (fig. 2) for either small $\Delta_{0}$ or small $U_{0}^{\prime}$ as caused by an optical gap which vanishes at either low- $\Delta_{0}$ or low$U_{0}^{\prime}$. Thus, we are interested in calculating the exciton-like parameters $A_{0}$ and $U_{0}^{\prime}$ (eqs. (2) and (1)) 

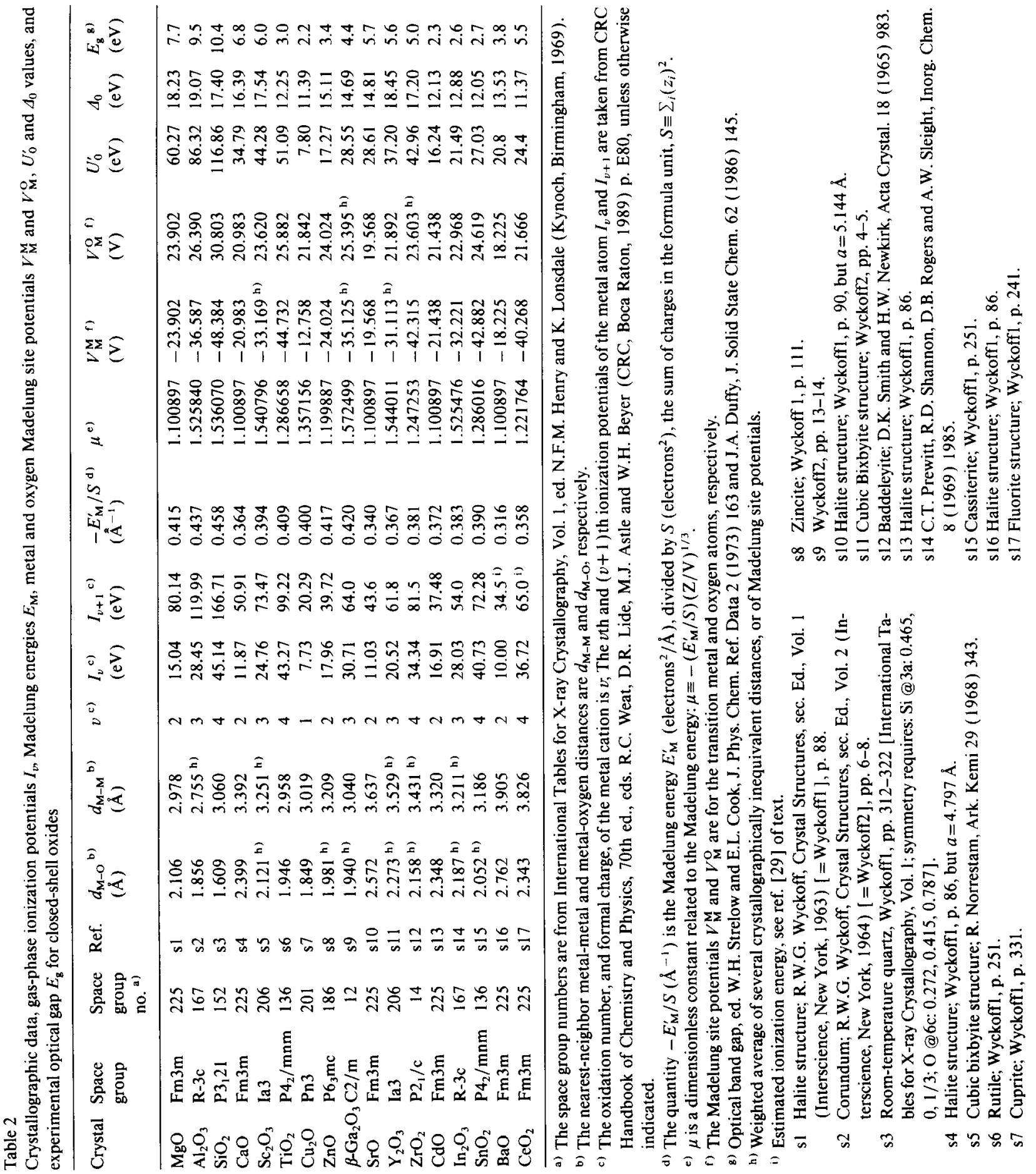
which are related to optical excitations in these oxides.

\section{Other oxides}

For the oxides in table 1, we show in table 3 the values of $\Delta V_{\mathrm{M}}, I_{v}, I_{v+1}, d_{\mathrm{M}-\mathrm{O}}$ and $d_{\mathrm{M}-\mathrm{M}}$ with the values $U_{0}^{\prime}$ and $\Delta_{0}$ calculated from eqs. (1) and (2). In figs. 4 and 5, we plot $U_{0}^{\prime}$ versus $\Delta_{0}$ for each of the simple and perovskite oxides, respectively $[25,26,29,37,38]$. The "insulators" and "metals" are shown as open and closed symbols, respectively, while the symbols with dots in the center represent those having metal-insulator transitions. It is clearly

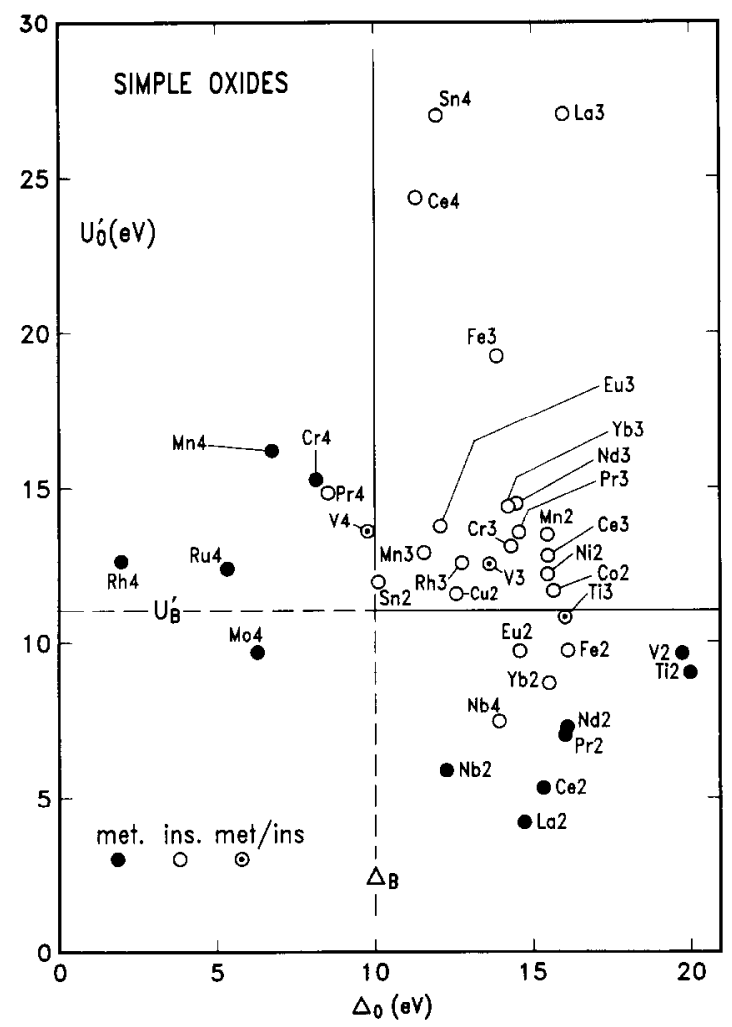

Fig. 4. A plot of the calculated values of $U_{0}^{\prime}$ and $\Delta_{0}$ for the 38 simple oxides in table 1 . The solid symbols represent the "metals" while the open symbols represent the "insulators". Those with a dot in the center exhibit a metal-insulator transition. The horizontal line at $U_{\mathrm{B}}$ and the vertical one at $\Delta_{\mathrm{B}}$ are drawn as boundaries to separate the "insulators" from the "metals", as in the ZSA diagram of fig. 2.

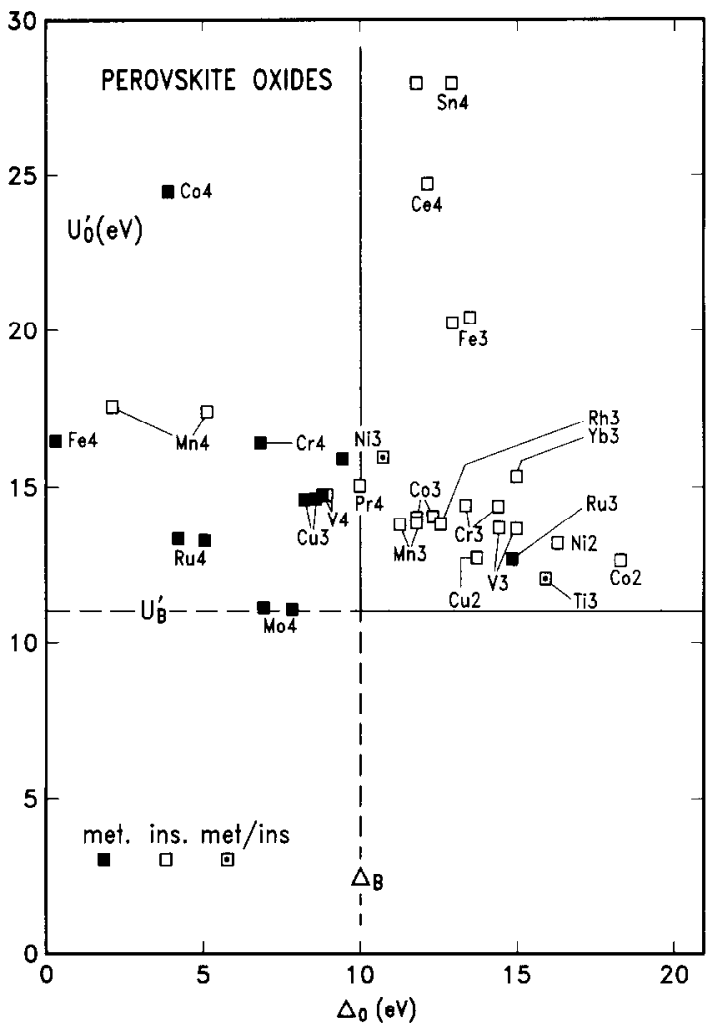

Fig. 5. A plot analogous to fig. 4 for the 38 perovskite-like oxides in table 1 .

seen [10] in figs. 4 and 5 that the "metallic" oxides tend to have either low values of $A_{0}$ or $U_{0}^{\prime}$. Attempting to put these data into the ZSA framework (as in fig. 2), one can separate most of the "insulators" from the "metals" by drawing a vertical and a horizontal boundary, such that the "metals" have either $\Delta_{0} \lesssim A_{\mathrm{B}}=10 \mathrm{eV}$ or $U_{0}^{\prime} \lesssim U_{\mathrm{B}}=11 \mathrm{eV}$. (We could achieve better separation if we chose a different $\Delta_{\mathrm{B}}$ and $U_{\mathrm{B}}$ for simple and perovskite oxides, but we want to keep things simple.) Thus, there are eight "metals" in figs. 4 and 5 which we can classify as low- $U^{\prime}$ metals and 16 "metals" which are classified as low$\Delta$ metals.

Another test of this model concerns those oxides in table 1 with metal-insulator transitions: these should fall on or near the boundary in fig. 2 between the "metals" and the "insulators". Indeed, we see in figs. 4 and 5 that all five compounds (shown by the symbols with a dot in their center) do lie near one 


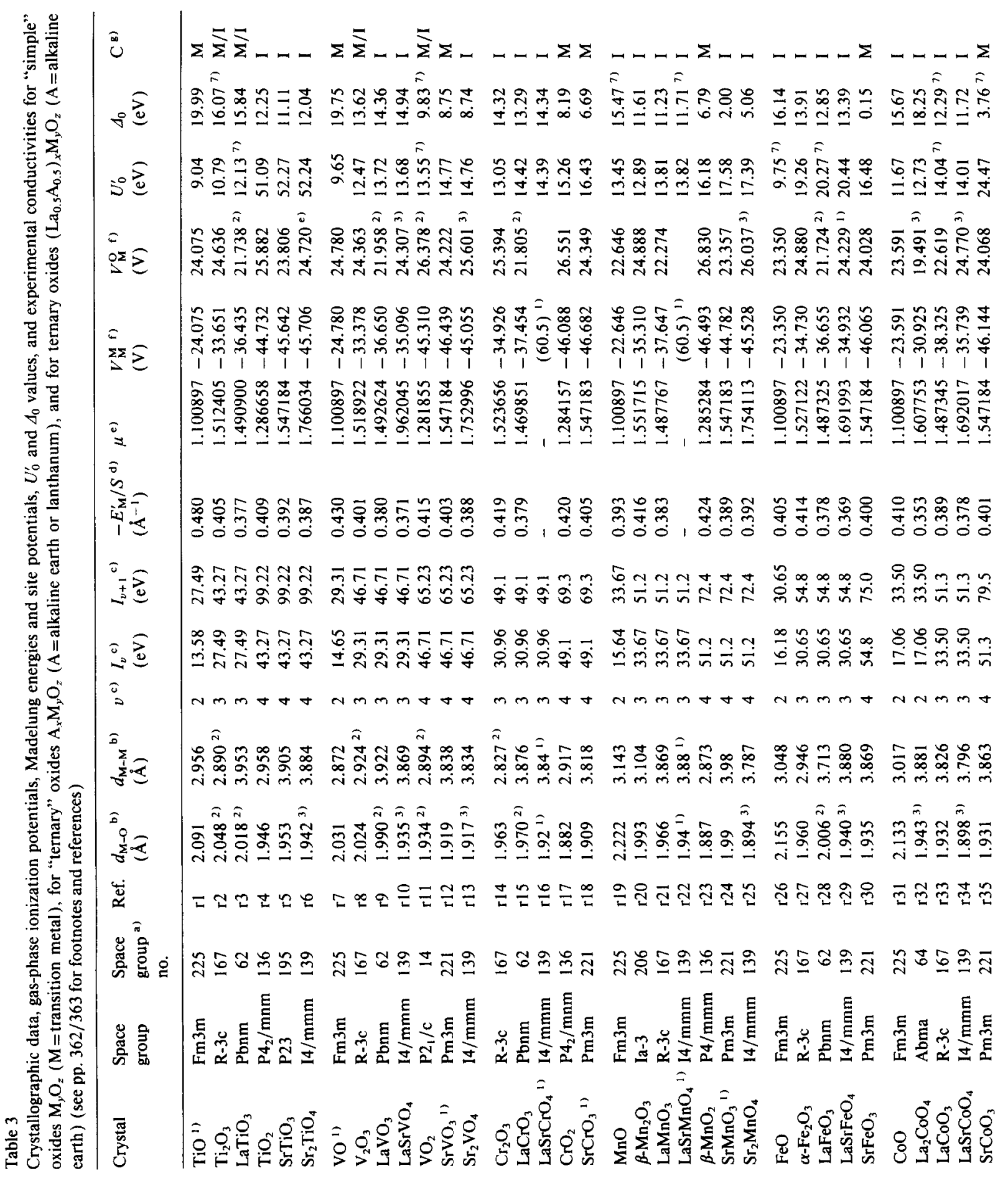




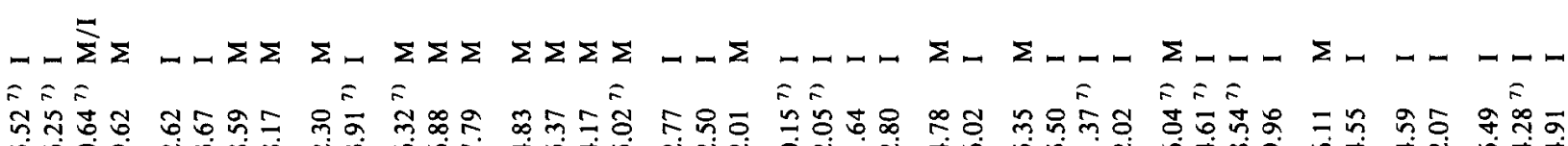
ம்ழல் సิำ

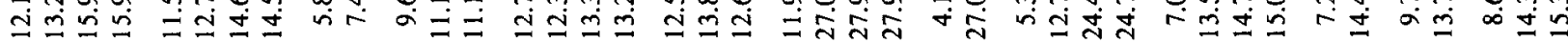
$=\hat{m} \hat{m} \hat{m} \quad \hat{N} \hat{m} \quad \hat{m} \quad \hat{m}$

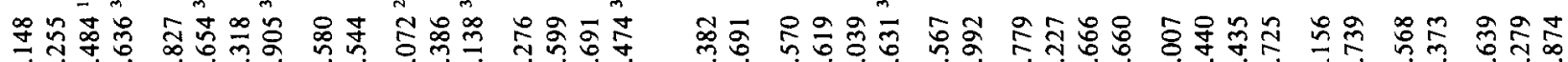

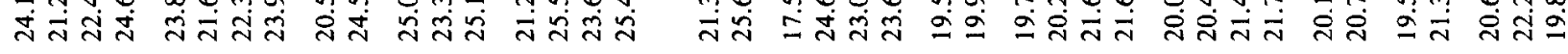

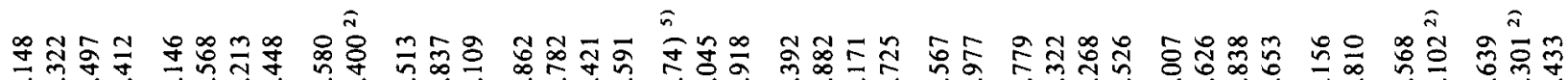

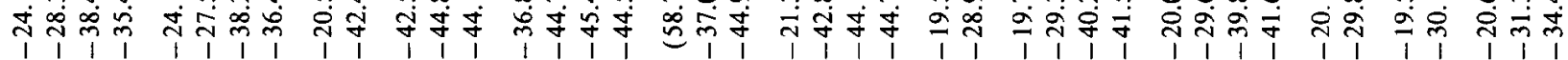

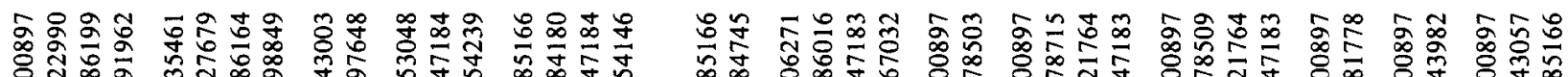

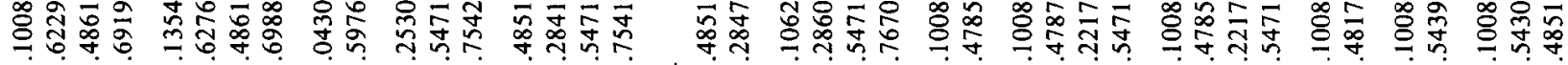

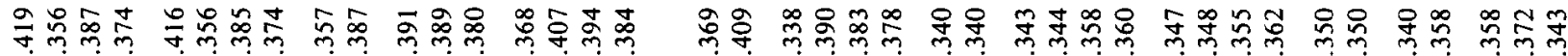

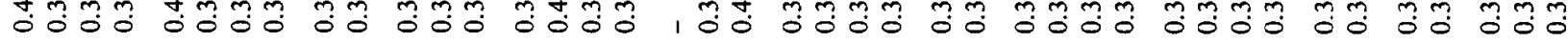
Iニa,

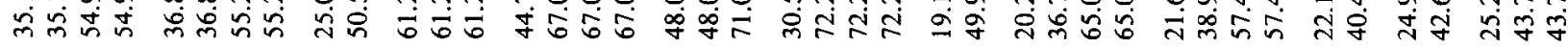

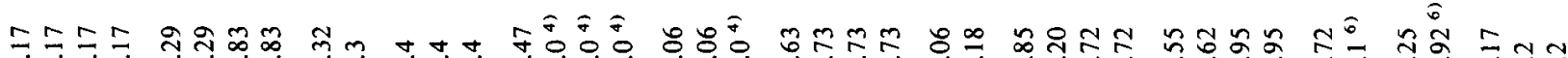

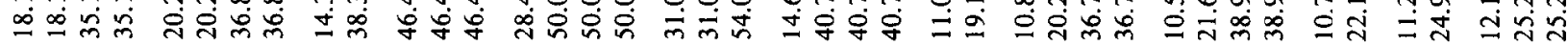
Namm nNmm ntg J J

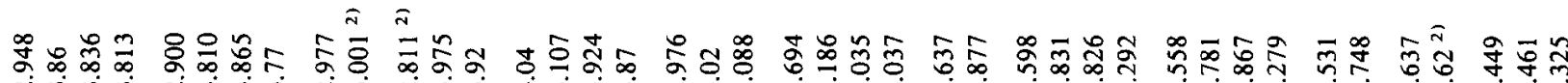
तmंm

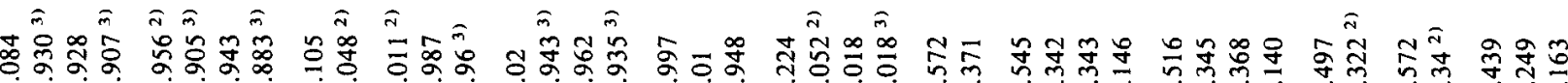
r-I- -

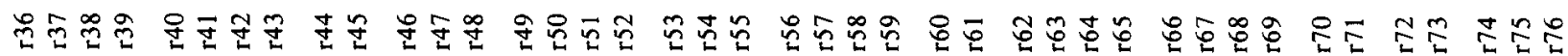

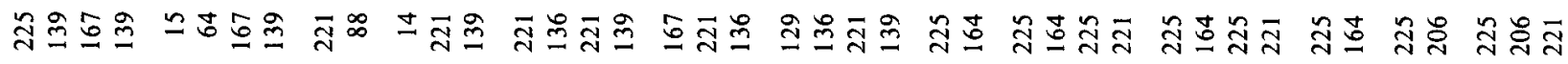

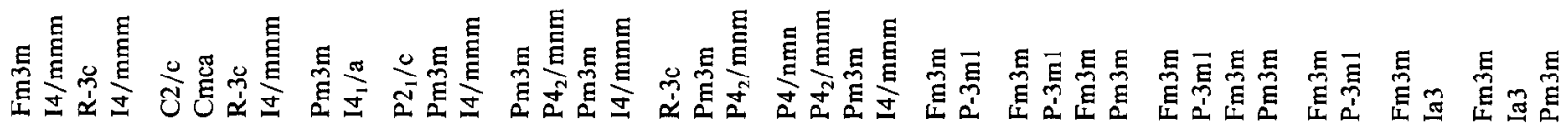

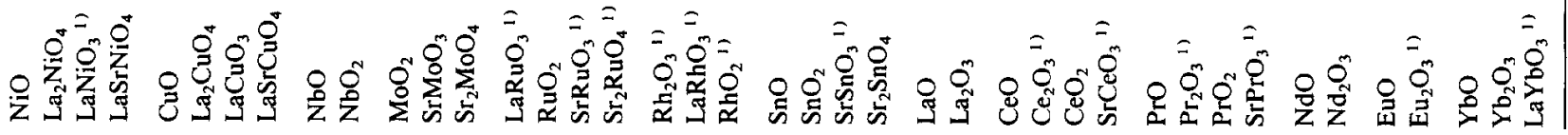


1) Approximate crystal structure.

2) Weighted average of several crystallographically inequivalent distances, or of several Madelung site potentials.

3) In-plane metal-oxygen distance (s) only.

4) Estimated ionization energy; where theoretical estimates had to supplant experimental data, since energy differences are relevant, in some cases, different values are used for the same ionization in different salts (e.g. $I_{4}=44.73 \mathrm{eV}$ for $\mathrm{LaRuO}_{3}$ but $I_{4}=49.9 \mathrm{eV}$ for $\mathrm{RuO}_{2}$ ); see also footnote 29 in text.

${ }^{5)}$ Madelung site potential difference $V_{\mathrm{M}}^{\mathrm{O}}-V_{\mathrm{M}}^{\mathrm{M}}$, from J.Q. Broughton and P.S. Bagus, J. Electron Spectr. Rel. Phenom. 20 (1980) 261, who probably used structural data from R.W.G. Wyckoff, Crystal Structures, sec. Ed., Vols. 1 and 2 (Interscience, New York, 1963, 1964).

6) W.C. Martin, L. Hagan, J. Reader and J. Sugar, J. Phys. Chem. Ref. Data 3 ( 1974) 771.

7) Because of new calculations, or different averages between sites, the $U_{0}^{\prime}$ and $\Delta_{0}$ values given here differ somewhat from those of J.B. Torrance, P. Lacorre, C. Asavaroengchai and R.M. Metzger, J. Solid State Chem. 90 (1991) 168.

a) The space group numbers are from International Tables for $\mathrm{X}$ ray Crystallography, either Vol. 1, eds. N.F.M. Henry and K. Lonsdale (Kynoch, Birmingham, UK, 1969) or Vol. A, ed. T. Hahn (Reidel, Dordrecht, Holland, 1983).

b) The nearest-neighbor transition metal-transition metal and transition metal-oxygen distances are $d_{\mathrm{M}-\mathrm{M}}$ and $d_{\mathrm{M}-\mathrm{O}}$, respectively.

c) The oxidation number, and formal charge, of the metal cation is $v$, The $v$ th and $(v+1)$ th ionization potentials of the metal atom $I_{\nu}$ and $I_{v+1}$ are taken from CRC Handbook of Chemistry and Physics, 70th Edition, eds. R.C. Weast, D.R. Lide, M.J. Astle and W.H. Beyer (CRC, Boca Raton, FL, 1989) p. E80, unless otherwise indicated.

d) The quantity $-E_{\mathrm{M}}^{\prime} / S\left(\AA^{-1}\right)$ is the Madelung energy $E_{\mathrm{M}}^{\prime}$ (electrons ${ }^{2} / \AA$ ), divided by $S$ (electrons ${ }^{2}$ ) $=$ the sum of charges in the formula unit, $S \equiv \sum_{i}\left(z_{i}\right)^{2}$; for instance, in $\mathrm{Ti}_{2} \mathrm{O}_{3}, S=30$ electrons"; $E_{\mathrm{M}}^{\prime} / S$ is remarkably "constant" from structure to structure. This trend was first reported by R.M. Metzger and J.B. Torrance, MRS Symp. Proc., Vol. 156 (Materials Research Society, Pittsburgh, PA, 1989), p. 377.

e) $\mu$ is a dimensionless constant related to the Madelung energy: $\mu \equiv-\left(E_{\mathrm{M}}^{\prime} / S\right)(Z / V)^{1 / 3}$. For $\mathrm{NaCl}$ (halite) $\mu=1.100897$, and $\mu 4^{1 / 3}=\alpha=1.747564$ yields the well-known binary Madelung "constant" $\alpha$ for the halite structure.

f) The Madelung site potentials $V_{M}^{\mathrm{M}}$ and $V_{\mathrm{M}}^{\mathrm{O}}$ are for the transition metal and oxygen atoms, respectively. When the Madelung site potentials were either estimated by extrapolation from other known structures, or ${ }^{5)}$ taken from J.Q. Broughton and P.S. Bagus, op. cit., then only the Madelung site potential difference $V_{M}^{O}-V_{M}^{M}$ is given in parentheses.

8) The entries under $C=$ Conductivity are: $M=$ metal, $M$ / $\mathrm{I}=$ borderline, or with semiconductor-to-metal transition, $\mathrm{I}=$ insulator or semiconductor).

r1 Halite structure; space group Fm3m (no. 225). R.W.G. Wyckoff, Crystal Structures, sec. Ed., Vol. 1 (Interscience, New York, 1963) [ = Wyckoff1 ], p. 90, but using $a=4.181 \AA$. There is a newer monoclinic structure, space group A2/m (no. 12), for TiO, by D. Watanabe et al., Acta Cryst. 23 (1967) 307; the $U_{0}^{\prime}$ values range from $2.57 \mathrm{eV}\left(d_{\mathrm{M}-\mathrm{M}}=2.54 \AA\right)$ to $13.91 \mathrm{eV}$ $\left(d_{\mathrm{M}-\mathrm{M}}=2.98 \AA\right)$; the $\Delta_{0}$ values range from 9.95 to $23.05 \mathrm{eV}$ $\left(V_{\mathrm{Ti}}=-18.188,-20.174\right.$, and $-25.844 \mathrm{~V}$ for Til, Ti3, and Ti2 respectively; $V_{\mathrm{O}}=18.188,20.174,25.844 \mathrm{~V}$ for O1, O3, and $\mathrm{O} 2$ respectively); for details see C. Asavaroengchai, Ph.D. dissertation, Univ. of Alabama, 1990.

r2 Corundum structure; R.W.G. Wyckoff, Crystal Structures, sec. Ed., Vol. 2 (Interscience, New York, 1964) [ = Wyckoff2], p. 6.

r3 D.A. MacLean, H.-N. Ng and J.E. Greedan, J. Solid State Chem. 30 (1979) 35.

r4 Rutile; Wyckoff1, p. 251.

r5 Cubic perovskite structure; G.W.C. Kaye and T.H. Laby, Tables of Physical and Chemical Constants, 14th ed. (Longman, London, 1973) p. 199.

r6 Tetragonal $\mathrm{K}_{2} \mathrm{MgF}_{4}$ structure; R.W.G. Wyckoff, Crystal Structurcs, scc. Ed., Vol. 3 (Interscience, New York, 1965) [=Wyckoff3], p. 69.

r7 Halite structure; cell constant for VO at $800^{\circ} \mathrm{C}$, Wyckoff 1 , p. 91.

r8 Corundum structure; Wyckoff2, p. 7.

r9 V.G. Zubkov, G.V. Baznev, V.A. Perelyaev and G.P. Shveikin, Sov. Phys. Solid State 15 (1973) 1079.

r10 J.M. Longo and P.M. Raccah, J. Solid State Chem. 6 ( 1973)

526. The LaSr site was assigned a formal charge of +2.5 .

rl1 Distorted rutile structure; Wyckoffl, p. 258; cell constants of D.B. Rogers, R.D. Shannon, A.W. Sleight and J.L. Gillson, Inorg. Chem. 8 (1969) 841 .

r12 B.L. Chamberland and P.S. Danielson, J. Solid State Chem. 3 (1971) 243.

rl 3 Tetragonal $\mathrm{K}_{2} \mathrm{MgF}_{4}$ structure; $\mathrm{M}$. Cyrot, B. Lambert-Andron, J.L. Soubeyroux, M.J. Rey, P.H. Dehaut, F. Cyrot-Lackmann, G. Fourcaudot, J. Beille and J.L. Tholence, J. Solid State Chem. 85 (1990) 321.

r14 Corundum structure; Wyckoff2, p. 6.

r1 5 C.P. Khattak and D.E. Cox, Mat. Res. Bull. 12 (1977) 463. r16 J.C. Joubert, A. Collomb, D. Elmaleh, G. Le Flem, A. Daoudi and G. Ollivier, J. Solid State Chem. 2 (1970) 343. The LaSr site was assigned to a formal charge of +2.5 .

r17 Rutile structure; W.H. Cloud, D.S. Schreiber and K.R. Babcock, J. Appl. Phys. 33 (1962) 1193.

r18 Assumed cubic perovskite structure; $a=3.818 \AA$. Note useful mathematical relations for the site potentials derived by F. Michel-Calendini and G. Mesnard, Comptes Rendus Acad. Sci. Paris, B II 267 (1968) 727.

r19 Halite structure; Wyckoff 1, p. 88.

r20 Cubic bixbyite structure; Wyckoff2, p. 5. Only the Mn1$O$ distances and $\mathrm{Mn} 1$ and $O$ site potentials are used.

r21 Formula is $\mathrm{La}_{0.94} \mathrm{Mn}_{0.98} \mathrm{O}_{3}$; B.C. Tofield and W.R. Scott, J. Solid State Chem. 10 (1974) 183.

r22 P. Ganguly and C.N. Rao, J. Solid State Chem. 53 (1984) 193. The LaSr site was assigned a formal charge of +2.5 .

r23 Cassiterite (tetragonal $\mathrm{SnO}_{2}$ ) structurc; Wyckoff 1, p. 251, but with new cell constants $a=4.3983 \AA, c=2.8730 \AA$, $u=0.3034$; note slightly different site potentials (Mn: -46.499 V, O: $26.833 \mathrm{~V}$ ) in J.Q. Broughton and P.S. Bagus, J. Electron Spectr. Rel. Phenom. 20 (1980) 261. 
r24 Assumed cubic perovskite structure; $a=3.98 \AA$. r25 Tetragonal $\mathbf{K}_{2} \mathbf{M g F}_{4}$ structure; J.-C. Bouloix, J.-L. Soubeyroux, G. Le Flem and P. Hagenmüller, J. Solid State Chem. 38 (1981) 34.

r26 Halite structure; Wyckoff1, p. 87.

r27 Corundum structure; Wyckoff2, p. 7.

r28 M. Marezio and P.O. Dernier, Mat. Res. Bull. 6 (1971) 23.

r29 J.-L. Soubeyroux et al., J. Solid State Chem. 31 (1980)

313. The LaSr site was assigned a formal charge of +2.5 .

r30 Cubic perovskite structure; Wyckoff2, p. 394.

r31 Halite structure; Wyckoff 1, p. 86.

r32 U. Lehmann and H. Müller-Buschbaum, Z. Anorg. Allgem. Chem. 470 (1980) 59.

r33 G. Thornton, B.C. Toficld and A.W. Hewat, J. Solid State Chem. 61 (1986) 301.

r34 G. Demazeau, P. Courbin, G. Le Flem, M. Pouchard, P. Hagenmüller, J.L. Soubeyroux, I.G. Main and G.A. Robins, Nouv. J. Chim. 3 (1979) 171. The LaSr site was assigned a formal charge of +2.5 .

r35 Assigned cubic perovskite structure; Wyckoff2, p. 394, but assigning $Z=1$ and $a=3.8625$.

r36 Halite structure; Wyckoff1, p. 89.

r37 $\mathrm{K}_{2} \mathrm{NiF}_{4}$ structure; Hk. Müller-Buschbaum and U. Lehmann, Z. Anorg. Allgem. Chem. 447 (1978) 47.

r38 G. Demazeau, C. Parent, M. Pouchard and P. Hagenmüller, Mat. Res. Bull. 7 (1972) 913.

r39 H. Pausch and H. Müller-Buschbaum, Z. Naturfosch. 34 B (1979) 378.

r40 Tenorite; S. Asbrink and L.J. Norby, Acta Cryst. B 26 (1970) 8 .

r41 B. Grande, H. Müller-Buschbaum and M. Schweitzer, Z. Anorg. Allgem. Chem. 428 (1977) 120.

r42 G. Demazeau, C. Parent, M. Pouchard and P. Hagenmüller, Mat. Res. Bull. 7 (1972) 913.

r43 J.B. Goodenough, G. Demazeau, M. Pouchard and P. Hagenmüller, J. Solid State Chem. 8 (1973) 325. The LaSr site was assigned a formal change of +2.5 .

r44 A.L. Bowman, T.C. Wallace, J.L. Yarnell and R.G. Wenzel, Acta Cryst. 21 (1966) 843; J.K. Burdett and T. Hughbanks, J. Am. Chem. Soc. 106 (1984) 3101.

r45 Distorted rutile structure, Wyckoffl, p. 260 , origin at -1 ; newer cell constants of D.B. Rogers, R.D. Shannon, A.W. Sleight and J.L. Gillson, Inorg. Chem. 8 (1969) 841.

r46 B.G. Brandt and A.C. Skapski, Acta Chem. Scand. 21 (1967) 661.

r47 Cubic perovskite structure; Wyckoff2, p. 394.

r48 Tetragonal $\mathrm{K}_{2} \mathrm{MgF}_{4}$ structure; Wyckoff3, p. 69; no $z(\mathrm{Sr})$ or $z(\mathrm{O} 2)$ listed; the approximate values $z(\mathrm{Sr})=0.356$, $z\left(\mathrm{O}_{2}\right)=0.157$ (from the $\mathrm{Sr}_{2} \mathrm{MnO}_{4}$ structure) were used here. r49 Assumed cubic perovskite structure; $a=4.04 \AA$. The actual structure is an orthorhombic distortion: R.J. Bouchard and J.F. Weiher, J. Solid State Chem. 4 (1972) 80.

r50 Rutile structure; C.-E. Boman, Acta Chem. Scand. 24 (1970) 116.

r51 Assumed approximate cubic perovskite structure (a $\mathrm{GdFeO}_{3}$ structure, space group Pbmn (no. 62) is assigned by
Wyckoff2, p. 409 , but $x(\mathrm{Sr}), y(\mathrm{Sr}), x(\mathrm{O} 1), y(\mathrm{O} 1), x(\mathrm{O} 2)$, $y(\mathrm{O} 2), z(\mathrm{O} 2)$ are not known ).

r52 Tetragonal $\mathrm{K}_{2} \mathrm{MgF}_{4}$ structure; Wyckoff3, p. 69 , no $z(\mathrm{Sr})$ or $z(\mathrm{O} 2)$ listcd; the approximate values $z(\mathrm{Sr})=0.356$, $z(\mathrm{O} 2)=0.157$ (from the $\mathrm{Sr}_{2} \mathrm{MnO}_{4}$ structure) were used here. r53 Corundum structure (approximate); Wyckoff2, p. 8 $(x(\mathrm{Rh}), x(\mathrm{O})$ not known).

r54 Assumed cubic perovskite structure; $a=4.02 \AA$. The actual structure is an orthorhombic distortion: R.J. Bouchard and J.F. Weiher, J. Solid State Chem. 4 (1972) 80.

r55 R.D. Shannon, Solid State Commun. 6 (1968) 139.

r56 J. Panettier and G. Denes, Acta Cryst. B 36 (1980) 2763. r57 Cassiterite; Wyckoff1, p. 251.

r58 Assumed cubic perovskite structure; $a=4.035 \AA$. Actual structure: A.J. Smith and A.J.E. Walsh, Acta Cryst. 13 (1960) 653.

r59 R. Weiss and R. Faivre, Compt. Rend. Acad. Sci. Paris 248 (1959) 106.

r60 Halite structure; J.M. Leger, N. Yacoubi and J. Loriers, J. Solid State Chem. 36 (1981) 261.

r61 Wyckoff2, p. 1 .

r62 Halite structure; J.M. Leger, N. Yacoubi and J. Loriers, J. Solid State Chem. 36 (1981) 261.

r63 Hexagonal $\mathrm{La}_{2} \mathrm{O}_{3}$ structure; Wyckoff2, p. 3 (approximate structure; $z(\mathrm{Ce})=0.245$ and $z\left(\mathrm{O}_{2}\right)=0.645$ were used, as in $\mathrm{La}_{2} \mathrm{O}_{3}$ ).

r64 Fluorite structure; Wyckoff1, p. 241.

r65 Cubic perovskite structure; M. Yoshimura, T. Nakamura and T. Sata, Chem. Lett. 1 (1973) 923.

r66 Halite structure; J.M. Leger, N. Yacoubi and J. Loriers, J. Solid State Chem. 36 (1981) 261.

r67 Hexagonal $\mathrm{La}_{2} \mathrm{O}_{3}$ structure; Wyckoff2, p. 3 (approximate structure: $z(\mathrm{Pr})=0.245$ and $z(\mathrm{O} 2)=0.645$ were used, as in $\left.\mathrm{La}_{2} \mathrm{O}_{3}\right)$.

r68 Fluorite structure; Wyckoffı, p. 243.

r69 Cubic perovskite structure; M. Yoshimura, T. Nakamura and T. Sata, Chcm. Lett. 1 (1973) 923.

r70 Halite structure; J.M. Leger, N. Yacoubi and J. Loriers, J. Solid State Chem. 36 (1981) 261.

r71 M. Faucher, J. Panettier, Y. Charriere and P. Caro, Acta Cryst. B 38 (1982) 344.

r72 H.A. Eick, N.C. Baenziger and L. Eyring, J. Am. Chem. Soc. 78 (1956) 5147.

r73 Cubic bixbyite structure, Wyckoff 2 , p. 5, with estimated $u(E u 2)=-0.03, x(\mathrm{O})=0.39, y(\mathrm{O})=0.15, z(\mathrm{O})=0.381$. For the more recent, precise monoclinic structure, space group $\mathrm{C} 2$ / m, H.L. Yakel, Acta Cryst. B 35 (1979) 564, the average Eu and $\mathrm{O}$ Madelung site potentials are $-30.185,21.104 \mathrm{eV}$, respectively.

r74 Halite structure; Wyckoff1, p. 91, but $a=4.877 \AA$ Å: J.M. Leger, J. Maugrion, L. Albert, J.C. Achard and C. Loriers, Compt. Rend. Acad. Sci. Paris 201 (1978) 2860.

r75 Cubic bixbyite structure; Wyckoff2, p. 4.

r76 Assumed cubic perovskite structure; $a=4.325 \AA$ at $2080^{\circ} \mathrm{C}$. J.P. Traverse, J. Coutures and M. Foex, Compt. Rend. Acad. Sci. Paris 267 (1968) 391. 
of these boundaries. This agreement provides strong independent support for this picture and further gives us information concerning the driving force for each of these metal-insulator transitions. For example, $\mathrm{Ti}_{2} \mathrm{O}_{3}, \mathrm{~V}_{2} \mathrm{O}_{3}$, and $\mathrm{LaTiO}_{3}$ each lie near the $U_{0}^{\prime}=$ $U_{\mathrm{B}}$ boundary, suggesting that the instability underlying the transition in these compounds involves low $U^{\prime}$, i.e., is related to the transfer of charge between metal cations. In fact, this is the Mott-Hubbard transition, which has been used [5] to describe the former two compounds, and is related to disproportionation. On the other hand, both $\mathrm{VO}_{2}$ and $\mathrm{LaNiO}_{3}$ fall near the $\Delta_{0}=A_{\mathrm{B}}$ boundary, implying that low- $\Delta$ or charge transfer excitations between oxygen and metal ions are responsible for the instability [15]. (We also note that, when off stoichiometry, both $\mathrm{FeO}$ and VO exhibit a metal-insulator transition [5] and both lie near the $U_{0}^{\prime}=U_{\mathrm{B}}$ boundary.)

The general success of this simple ionic model is evident in figs. 3, 4 and 5. There is a correlation (fig. 3 ) of the gap with $\Delta_{0}$ for the closed-shell cation oxides. In the ZSA diagram (figs. 4 and 5), boundaries at $U_{\mathrm{B}}$ and $\Delta_{\mathrm{B}}$ can be drawn which give a reasonable separation between "metals" and "insulators", with the compounds exhibiting metal-insulator transitions lying near these boundaries. This general agreement is strong evidence for the validity of such an ionic model as a first starting point to describe the electronic structure of oxides. Of course, there are discrepancies and disagreements also evident: "noise" of 1-2 eV and some oxides which are "exceptions". These are an indication and a measure of the role and significance of the approximations made.

(1) We have assumed that the electronic and lattice polarization effects are the same for all the oxides. As discussed above, the magnitude of these effects should reduce $\Delta_{0}$ and $U_{0}^{\prime}$ by $\sim 4 \mathrm{eV}$. It is expected that this screening energy will, in fact, not be the same for all oxides.

(2) We have assumed that the covalent and crystal field effects are the same for all oxides in table 1 . Stronger covalent effects are presumably responsible for the three outlying compounds in fig. 3 , as discussed above.

(3) A third simplifying assumption was that the bandwidth, $W$, is the same for all three bands in fig. 1 and for all oxides. This is clearly not the case. For example, the bandwidth for rare earth oxides is less than the first series transition-metal oxides, perhaps explaining why "insulating" $\mathrm{EuO}, \mathrm{YbO}, \mathrm{PrO}_{2}$, and $\mathrm{SrPrO}_{3}$ are calculated to lie in the metallic regions in figs. 4 and 5. In addition, there are two other major factors influencing the magnitude of $W$ : dimensionality and distortion. The two-dimensional $\mathrm{K}_{2} \mathrm{NiF}_{4}$ structures have $\mathrm{a} \sim 33 \%$ narrower band than their three-dimensional cubic perovskite counterparts [36]. Presumably this effect accounts for the insulating behavior of $\mathrm{Sr}_{2} \mathrm{VO}_{4}$ compared with metallic $\mathrm{SrVO}_{3}$. As an example of the effect of distortion, the calculated values of $U_{0}^{\prime}$ and $\Delta_{0}$ in the series $\mathrm{REMO}_{3}$, are approximately the same for each of the rare earths. However, the distortions of the perovskite structure increase considerably as the rare earth radius decreases, giving rise to a decreasing $W$. Presumably [15], this is the factor responsible for the insulating behavior of $\mathrm{YNiO}_{3}$ and $\mathrm{YTiO}_{3}$ compared with metallic $\mathrm{LaNiO}_{3}$ and $\mathrm{LaTiO}_{3}$.

Presumably, breakdown of these assumptions is responsible for the $\sim 1-2 \mathrm{eV}$ "noise" evident in figs. 3-5. However, outside of this "noise", there are a few additional exceptions: $\mathrm{NbO}_{2}$ is predicted to be a low $U_{0}^{\prime}$ metal and yet it has low conductivity at 300 $\mathrm{K}$. This fact is undoubtedly related to the observed distortion which pairs neighboring $\mathrm{Nb}$ atoms, since $\mathrm{NbO}_{2}$ becomes metallic near $850^{\circ} \mathrm{C}$ when this distortion disappears [4]. $\mathrm{SrMnO}_{3}$ and $\mathrm{Sr}_{2} \mathrm{MnO}_{4}$ are predicted to be low $\Delta_{0}$ metals (with very low $\Delta_{0}$ ), but are observed to be insulating. We have no explanation for this fact.

Historically, the dcscription $[4,6,7]$ of the electronic properties of oxides has been aided by the framework of Goodenough [4], in which the physical properties are largely determined by the value of the transfer energy, $b$. For example, metallic conductivity has been correlated with a large transfer energy, which is indicated by lattice parameters which are short compared with the sum of the ionic radii. What was not clear, however, was what caused the differences in transfer energy between compounds. We suggest that the short lattice constants (metallic bonding), large transfer energies, and metallic conductivity are all caused by and are all consequences of something else: either a small value of $U_{0}^{\prime}$ or small $A_{0}$. 


\section{Conclusion}

It is clear that this simple ionic model of oxides is only a starting point, although a remarkably good one. There are a number of areas needing modification and improvement. We know that the electronic overlap is large and can have important consequences $[35,36,39]$. Similarly, the electronic and lattice polarization energies are large $[33,40]$ and there will be some consequences of the crystal field splittings, etc. Some of these effects probably will need to be included before this model can be extended to the interesting oxides of the third transition series (e.g., $\mathrm{Pb}, \mathrm{Tl}, \mathrm{Bi}$, etc.) and to the sulfides and selenides. One approach which we suggest is to start with the energy levels of the ionic model (which correctly include the important correlation effects ) and add perturbatively the effect of electronic overlap.

In conclusion, we have examined the conductivity behavior of a large variety of simple and perovskite oxides, including 24 "metals", 47 "insulators" and 5 compounds undergoing metal-insulator transitions. Using a simple ionic model in which we include only the electrostatic Coulomb interactions between the ions and the gas phase ionization potentials, we can reasonably account for most of these trends, as well as for the band gaps for 14 closedshell metal oxides. The breadth of this agreement gives confidence that this simple ionic model is a remarkably good starting point for understanding the electronic structure of oxides.

\section{References}

[1] A.W. Sleight, J.L. Gillson and P.E. Bierstedt, Solid State Commun. 17 (1975) 25.

[2] J.G. Bednorz and K.A. Müller, Z. Phys. B 64 (1986) 189.

[3] R.J. Cava, B. Batlogg, J.J. Krajewski, R. Farrow and L.W. Rupp, Nature 332 (1988) 814.

[4] J.B. Goodenough and J.M. Longon, Landolt-Börnstein Tabellen, Vol. 111/4a (Springer, Berlin, 1970);

S.Nomura, Landolt-Börnstein Tabellen Vol. 111/12a (Springer, Berlin, 1978);

J.B. Goodenough, Progr. Solid State Chem. 5 (1971) 149.

[5] D. Adler, Radiation Effects 4 (1970) 123, and references therein.

[6] C.N.R. Rao and G.V. Subbarao, Phys. Status Solidi A 1 (1970) 597;

C.N.R. Rao, Ann. Rev. Phys. Chem. 40 (1989) 291, and references therein.
[7] M. Pouchard et al., Mat. Res. Symp. Proc. 156 (1989) 13, and references therein.

[8] "The Oxide Handbook", ed. G.V. Samsonov (IFI/Plenum, New York, 1982).

[9] A.W. Sleight, Proc. Welch Conf. on Valency 123 (1989), and references therein.

[10] For a preliminary account of this work, see $\mathbf{J}$. Torrance, $\mathbf{P}$. Lacorre, C. Asavaroengchai and R.M. Metzger, J. Solid State Chem. 90 (1991) 168.

[11] We have not included in table 1 oxides from the third row of the transition series because their orbitals tend to be so delocalized that a simple ionic model (such as we shall use later) might not apply.

[12 ] H.K. Bowen, D. Adler and B.H. Auker, J. Solid State Chem. 12 (1975) 355.

[13] W.H. Strehlow and E.L. Cook, J. Phys. Chem. Ref. Data 2 (1973) 163.

[14] D.A. MacLean and J.E. Greedan, Inorg. Chem. 20 (1981) 1025.

[15] P. Lacorre, J.B. Torrance, J. Pannetier, A.I. Nazzal, P.W. Wang and T.C. Huang, J. Solid State Chem. 91 (1991) 225.

[16] Such transitions are sometimes also called "metalnonmetal" or "metal-semiconductor" transitions. Due to the dramatic change in conductivity observed in the cases discussed here, we refer to the transitions as "metalinsulator".

[17] J. Zaanen, G.A. Sawatzky and J.W. Allen, Phys. Rev. Lett. 55 (1985) 418;

G.A. Sawatzky and J.A. Allen, Phys. Rev. Lett. 53 (1984) 2339.

[18] J. Zaanen and G.A. Sawatzky, J. Solid State Chem. 88 (1990) 8, and references therein.

[19] Our values are related to those often used by theorists by the following relations: $U_{0}^{\prime}=U_{\mathrm{d}}-e^{2} / d_{\mathrm{M}-\mathrm{M}}$ and $\Delta_{0}=\epsilon_{\mathrm{p}}-\epsilon_{\mathrm{d}}+e^{2} / d_{\mathrm{M}-\mathrm{o}}$

[20] M. O'Keeffe and M. Valigi, J. Phys. Chem. Solids 31 (1970) 310.

[21 ] P.D. Citrin and T.D. Thomas, J. Chem. Phys. 57 (1972) 4446.

[22] J.B. Torrance and R.M. Metzger, Phys. Rev. Lett. 63 (1989) 1515.

[23] J. Kondo, J. Phys. Soc. Jpn. 58 (1989) 2884.

[24] Y. Ohta, T. Tohyama and S. Mackawa, Physica C 166 (1990) 385.

[25] In layered structures $\left(\mathrm{La}_{2} \mathrm{MO}_{4}, \mathrm{LaSrMO}\right.$, and $\left.\mathrm{Sr}_{2} \mathrm{MO}_{4}\right)$, $\Delta V_{\mathrm{M}}$ is calculated for excitation to the oxygen in the plane (not the apical oxygen). For other cases when there are inequivalent oxygen sites (e.g., distorted $\mathrm{LaMO}_{3}$ ), the averaged $\Delta V_{\mathrm{M}}$ is tabulated.

[26] In the case where there are two inequivalent metal sites, there would also be a $\Delta V_{M}$ term in eq. (1) but this is negligible or zero in the cases of interest.

[27] J.Q. Broughton and P.S. Bagus, J. Electr. Spectr. Rel. Phenom. 20 (1980) 261.

[28] R.M. Metzger, J. Chem. Phys. 57 (1972) 1870; ibid., J. Chem. Phys. 64 (1976) 2069. 
[29] When the experimental values are unknown ( $I_{4}$ for $\mathrm{Ru}$ and $\mathrm{Rh} ; I_{5}$ for $\mathrm{Ce}, \mathrm{Ru}$ and $\mathrm{Rh}$ ), the theoretical values (Carlton et al., Atomic Data 2 (1970) 63) are used. This can be a serious source of errors, since the theoretical value for $I_{3}$ for $\mathrm{Ru}$, for example, is $5 \mathrm{eV}$ larger than the experiment. In an attempt to minimize such errors, we use the difference between the two theoretical values of $I_{4}$ and $I_{3}$ to calculate $U_{0}^{\prime}$ for $\mathrm{LaRuO}_{3}, \mathrm{LaRhO}_{3}$ and $\mathrm{Rh}_{2} \mathrm{O}_{3}$, and use the experimental value for $I_{3}$ to calculate $\Delta_{0}$.

[30] "CRC Handbook of Chemistry and Physics" (CRC, Boca Raton, 1989), p. E80;

W.C. Martin, L. Hagan, J. Reader and J. Sugar, J. Phys. Chem. Ref. Data 3 (1974) 771.

[31 ] M. O'Keeffe, J. Solid State Chem. 85 (1990) 108.

[32] J.A. Duffy, J. Solid State Chem. 62 (1986) 145.

[33] M.S. Islam, M. Leslie, S.M. Tomlinson and C.R.A. Catlow, J. Phys. C 21 (1988) L109.
[34] The electronic and total screening of a $U^{\prime}$-like $\left(\mathrm{Cu}^{3+}+\mathrm{Cu}^{+}\right)$ excitation are calculated [33] to be 6.5 and $11.8 \mathrm{eV}$, respectively, and for a 4 -like $\left(\mathrm{O}^{-}+\mathrm{Cu}^{+}\right)$excitation are 5.4 and $11.1 \mathrm{eV}$.

[35] W.E. Pickett, Rev. Mod. Phys. 61 (1989) 433, and references therein.

[36] L.M. Mattheiss, Phys. Rev. Lett. 58 (1987) 1028; Phys. Rev. B 42 (1990) 359 .

[37] Data for $\mathrm{TiO}_{2}, \mathrm{SrTiO}_{3}$ and $\mathrm{Sr}_{2} \mathrm{TiO}_{4}$ cannot be seen in figs. 4 or 5 because their value of $U_{0}^{\prime}$ is too large.

[38] For TiO and VO, $\Delta V_{\mathrm{M}}$ is calculated for the average rock salt structure.

[39] J.K. Burdett and G.V. Kulkarni, Phys. Rev. B 40 (1989) 8908.

[40] N.L. Allen, J.M. Lawton and W.C. Mackrodt, J. Phys. 1 (1989) 2657;

R.C. Baetzold, Phys. Rev. B 42 (1990) 56, and references therein. 